\title{
Fatigue and perceptual responses of heavier- and lighter-load isolated lumbar extension resistance exercise in males and females
}

\author{
Charlotte Stuart $^{1}$, James Steele ${ }^{1}$, Paulo Gentil ${ }^{2}$, Jürgen Giessing ${ }^{3}$, James P Fisher ${ }^{\text {Corresp. }}{ }^{1}$ \\ ${ }^{1}$ School of Sport, Health and Social Sciences, Southampton Solent University, United Kingdom \\ 2 Faculty of Physical Education and Dance, Universidade Federal de Goiás, Brazil \\ 3 Institute of Sport Science, University of Koblenz-Landau, Germany \\ Corresponding Author: James P Fisher \\ Email address: james.fisher@solent.ac.uk
}

Background: There is a lack of research considering acute fatigue responses to high- and low-load resistance training as well as the comparison between male and female responses. Furthermore, limited studies have considered fatigue response testing with the inclusion of perceptions of discomfort and exertion. Methods: The present study included males $(n=9 ; 23.8 \pm 6.4$ years; $176.7 \pm 6.2 \mathrm{~cm} ; 73.9 \pm 9.3 \mathrm{~kg})$ and females $(n=8 ; 21.3$ \pm 0.9 years; $170.5 \pm 6.1 \mathrm{~cm} ; 65.5 \pm 10.8 \mathrm{~kg}$ ) who were assessed for differences in fatigue (i.e. loss of torque at maximal voluntary contraction (MVC)) immediately following isolated lumbar extension (ILEX) exercise at heavy- (HL) and light-(LL) loads ( $80 \%$ and $50 \%$ MVC, respectively). Participants also reported perceptual measures of effort (RPE-E) and discomfort (RPE-D) between different resistance training protocols. Results: Analysis of variance revealed significantly greater absolute and relative fatigue following LL compared to $\mathrm{HL}$ conditions $(p<0.001)$. Absolute fatigue significantly differed between males and females ( $p=0.012)$, though relative fatigue was not significantly different $(p=0.160)$. However, effect sizes for absolute fatigue $(\mathrm{HL}$; Males $=-1.84$, Females $=-0.83$; $\mathrm{LL}$; Males $=$ -3.11 , Females $=-2.39)$ and relative fatigue $(\mathrm{HL}$; Males $=-2.17$, Females $=-0.76$; $L \mathrm{~L}$; Males $=-3.36$, Females $=-3.08$ ) were larger for males in both HL and LL conditions. RPE-E was maximal for all participants in both conditions, but RPE-D was significantly higher in LL compared to HL $(p<0.001)$ with no difference between males and females. Discussion: Our data suggests that females do not incur the same degree of fatigue as males following similar exercise protocols, and indeed that females might be able to sustain longer exercise duration at the same relative loads. As such females should manipulate training variables accordingly, perhaps performing greater repetitions at a relative load, or using heavier relative loads than males. Furthermore, since lighter load exercise is often prescribed in rehabilitation settings (particularly for the lumbar extensors) it seems 
prudent to know that this might not be necessary to strengthen musculature and indeed might be contraindicated to avoid the increased fatigue and discomfort associated with LL exercise. 
1 Title: Fatigue and perceptual responses of heavier- and lighter-load isolated lumbar extension resistance

2 exercise in males and females

3

4 Running title: Lumbar fatigue in males and females

5

6 Original Research Article

7

8 Charlotte Stuart $^{1}$, James Steele ${ }^{1}$, Paulo Gentil ${ }^{2}$, Jürgen Giessing ${ }^{3}$, James P. Fisher ${ }^{1}$

9

${ }^{1}$ School of Sport, Health and Social Sciences, Southampton Solent University, United Kingdom

$11{ }^{2}$ Faculty of Physical Education and Dance, Federal University of Goias, Brazil

$12{ }^{3}$ Institute of Sport Science, University of Koblenz-Landau, Germany

13

14 Corresponding Author:

15 James P. Fisher

16 School of Sport, Health and Social Sciences

17 Southampton Solent University

18 United Kingdom

19 Tel: +442382013163

20 Email: James.Fisher@solent.ac.uk

21

22

Co-Author contact details:

23

Charlotte Stuart (charlotte.stuart95@outlook.com)

24 James Steele (James.Steele@solent.ac.uk)

25 Paulo Gentil (paulogentil@hotmail.com)

26 Jürgen Giessing (giessing@uni-landau.de)

27

28

Word Count: 4661 


\section{Abstract}

Background: There is a lack of research considering acute fatigue responses to high- and low-load

resistance training as well as the comparison between male and female responses. Furthermore, limited studies have considered fatigue response testing with the inclusion of perceptions of discomfort and exertion. Methods: The present study included males ( $n=9 ; 23.8 \pm 6.4$ years; $176.7 \pm 6.2 \mathrm{~cm} ; 73.9 \pm 9.3 \mathrm{~kg}$ ) and females ( $n=8 ; 21.3 \pm 0.9$ years; $170.5 \pm 6.1 \mathrm{~cm} ; 65.5 \pm 10.8 \mathrm{~kg}$ ) who were assessed for differences in fatigue (i.e. loss of torque at maximal voluntary contraction (MVC)) immediately following isolated lumbar extension (ILEX) exercise at heavy- (HL) and light-(LL) loads ( $80 \%$ and $50 \%$ MVC, respectively). Participants also reported perceptual measures of effort (RPE-E) and discomfort (RPE-D) between different resistance training protocols. Results: Analysis of variance revealed significantly greater absolute and relative fatigue following LL compared to HL conditions $(p<0.001)$. Absolute fatigue significantly differed between males and females $(p=0.012)$, though relative fatigue was not significantly different $(p=0.160)$. However, effect sizes for absolute fatigue (HL; Males $=-1.84$, Females $=-0.83 ; \mathrm{LL} ;$ Males $=-3.11$, Females $=-2.39)$ and relative fatigue $(\mathrm{HL} ;$ Males $=-2.17$, Females $=-0.76 ; \mathrm{LL}$; Males $=-3.36$, Females $=-3.08$ ) were larger for males in both $\mathrm{HL}$ and $\mathrm{LL}$ conditions. RPE-E was maximal for all participants in both conditions, but RPE-D was significantly higher in LL compared to HL ( $p<$ 0.001) with no difference between males and females. Discussion: Our data suggests that females do not incur the same degree of fatigue as males following similar exercise protocols, and indeed that females might be able to sustain longer exercise duration at the same relative loads. As such females should manipulate training variables accordingly, perhaps performing greater repetitions at a relative load, or using heavier relative loads than males. Furthermore, since lighter load exercise is often prescribed in rehabilitation settings (particularly for the lumbar extensors) it seems prudent to know that this might not be necessary to strengthen musculature and indeed might be contraindicated to avoid the increased fatigue and discomfort associated with LL exercise. 
54

55

56

57

58

59

60

61

62

63

64

65

\section{Introduction}

5

Strengthening of the lumbar extensors using isolated lumbar extension (ILEX) resistance exercise

is evidenced to be achievable using a low-volume (single-set) and low-frequency ( 1 day / week)

approach in both asymptomatic trained males (Fisher, Bruce-Low \& Smith, 2013; Steele, et al., 2015b)

and males and females symptomatic of chronic low back pain (CLBP; Bruce-Low et al., 2012; Steele et al., 2015a). A growing body of research supports that deconditioning of the lumbar extensor musculature

(e.g. decreased strength and greater fatigability) are factors associated with CLBP (Steele et al., 2014). As

such, strengthening of the lumbar extensors, where isolation is achieved using a restraint system

preventing rotation of the pelvis, has been recommended to condition (i.e. strengthen, improve

fatigability) this muscle group (Steele et al., 2013; Gentil et al., 2017b), and has been shown to reduce

CLBP (Bruce-Low et al., 2012; Steele et al., 2015a).

A definition of fatigue presented, and generally accepted, in the literature is "an acute impairment of performance that includes both an increase in the perceived effort necessary to exert a desired force and the eventual inability to produce this force" (Enoka \& Stuart, 1992; page 1631). From

this it has been hypothesised that the rate of muscle fatigue should coincide with the force requirements of the specific task to equate to a $100 \%$ value (Morton, McGlory \& Phillips, 2015). For example, if exercise were performed at $\sim 70 \%$ MVC to momentary failure this would produce $~ 30 \%$ muscle fatigue, or if exercise was performed to momentary failure using 30\% MVC then this would incur $70 \%$ fatigue. However, whilst research has suggested that greater fatigue is evident following lighter- compared to heavier-load exercise (30\% vs. $80 \%$ MVC, respectively; Fisher, Farrow \& Steele, 2017a), the relationship appears more complex than simply $100 \%$ minus the force requirements of the task relative to MVC. For example, in the study by Fisher, et al. exercise to momentary failure at $30 \%$ MVC produced a decrement in force production of $37.94 \%$, which was greater than the $13.48 \%$ reduction after exercise using $80 \%$ MVC. In addition, when effort matched to task failure (confirmed by 
maximal values using rating of perceived exertion; RPE), lighter load exercise appears to produce a greater degree of discomfort compared to heavier-load exercise (Fisher, Ironside \& Steele, 2017b). However, these studies have considered the knee extensors and as such cannot be used to infer a relationship for the lumbar extensors. Further, they have also only considered male participants.

Whilst there is a growing body of literature comparing fatigability and muscular endurance between males and females, the data appears equivocal when considering different muscle groups as well as muscle action performed. For example, Hunter, et al. (2004) reported similar fatigability of the elbow flexors when measured by time to task failure for a sustained submaximal isometric contraction (20\% maximal voluntary contraction; MVC) for strength matched males and females. However, Gentil, et al. (2017a), when comparing trained and untrained males and females, recently reported that females demonstrate a higher fatigue tolerance, when considering percentage reduction in isokinetic torque, than males of a similar training status when performing elbow flexion exercise. Maughan, et al. (1986) has reported significantly greater time to failure for females compared to males at lighter- but not heavier- loads ( $20 \%$, but not $50 \%$ or $80 \%$ MVC for isometric knee extension; and $50 \%, 60 \%$ and $70 \%$, but not $80 \%$ or $90 \%$ 1-repetition maximum for dynamic elbow flexion).

Considering the lumbar and trunk extensors specifically, Clark et al. (2003) reported similar muscular endurance performance for males and females for a dynamic trunk extension (TEX) task (women $=24.3 \pm 3.4$, men $=24.0 \pm 2.8$ repetitions at $50 \% \mathrm{MVC}$ ). However, when performing an isometric endurance test (time to task failure at $50 \% \mathrm{MVC}$ ), males demonstrated a briefer time to failure than females (105.4 \pm 7.9 compared to $146.0 \pm 10.9$ seconds, respectively). The authors also reported a similar fatigue response (reported as median frequency slope using electromyography; EMG) between the lumbar extensors and the biceps femoris for females, whereas males showed a greater fatigue in the lumbar extensors than the biceps femoris. However, our own laboratory has recently reported no relationship between TEX endurance using the Biering-Sorensen test (as used by Clark et al.) and ILEX 
102 strength both for asymptomatic- and CLBP symptomatic- participants (Conway et al., 2016). As such

103 there seems disparity between dynamic and isometric exercise and testing methods between males and

104 females. Furthermore, the current body of research cannot be applied specifically to the fatigability of

105 the lumbar extensor musculature in isolation.

106 Combined, improved understanding of the fatigability of the lumbar extensors, as well as the

107 associated discomfort, in response to different loading strategies and the impact of sex would be useful

108 in the prescription of sex specific exercise. At present recovery from fatiguing exercise from different

109 loading strategies in men and women is not well explored, and the sex-based differences in fatigability

110 may be of importance to determine optimal strategies for training and rehabilitation of the lumbar

111 musculature (Hunter, 2016). As such the aims of the present study were to compare the fatigue

112 responses of participants performing ILEX resistance exercise at heavier- and lighter- loads as well as

113 compare these between male and female participants.

114 Methods

115 Experimental approach to the Problem

116 The relationship between, load, sex, and fatigue response was examined across 3 testing

117 conditions using an ILEX machine (MedX, Ocala, Florida). A fatigue response test (FRT) was performed

118 for the following conditions; heavy load (HL; 80\% MVC), light load (LL; 50\% MVC) and control (CON; no

119 training). The conditions were separated by no less than 72 hours, with the condition type (HL, LL, CON)

120 being randomized to minimize order effect.

121 Participants

122 Approval was granted from the University Health, Exercise, and Sport Science (HESS) ethics

123 committee at the first authors' institution (ID No. 687). Previous work from our group using knee 
124 extension revealed a between-condition effect size (ES) of 1.86 (between HL and LL). Sample estimate

125 was based upon this to determine participant numbers $(n)$ calculated using G*Power (Faul et al., 2007;

126 Faul et al., 2009). The calculations showed that a minimum of 4 participants were necessary to meet the

127 required power of 0.8 at an alpha level of $p<0.05$ for comparison between loading conditions. However,

128 recruitment for each sex group (males and females) was increased to $n=10$ to provide greater power for

129 secondary analysis of between-sex comparisons. Recreationally active asymptomatic males $(n=10)$ and

130 females $(n=10)$ with no previous training experience of the lumbar extensors were recruited (see Table 1

131 for characteristics). Prior to testing, participants completed an informed consent and a physical activity

132 readiness questionnaire (PARQ). For the purpose of this study, the exclusion criteria included:

133 individuals who suffered from a heart condition, history of CLBP, any contraindications to exercise

134 identified on the participant PARQ, or any knee or hip conditions which prevented the use of the

135 machine restraints.

\section{Testing procedures}

Prior to testing, all participants attended a familiarization session where they were assessed for

lumbar range of motion using a goniometer built in to the ILEX machine, and performed isometric

testing using the ILEX machine every $12^{\circ}$ beginning at $72^{\circ}$ (full lumbar flexion) through $60^{\circ}, 48^{\circ}, 36^{\circ}, 24^{\circ}$,

$12^{\circ}$, and $0^{\circ}$ (full lumbar extension) to allow them to experience the technique required and reduce any

141 learning effect. Details of the full test protocol using the MedX ILEX machine and its restraint system

142 have been documented elsewhere (Graves et al., 1990). In brief, participants were provided a specific

143 dynamic warm-up of the lumbar extensors for $\sim 60$ seconds using $\sim 27 \mathrm{~kg}$ for males and $\sim 20 \mathrm{~kg}$ for females.

144 An MVC was then performed at 7 different joint angles (as described above) by the participant gradually

145 building force up to maximal effort over 3 seconds, and then relaxing over a further 3 seconds.

146 Participants were provided with verbal encouragement to ensure maximal effort and were permitted

$147 \sim 10$ seconds recovery between testing angles. The ILEX machine and restraint system is shown in figure 
1481 and prevents rotation of the pelvis allowing training and testing of the lumbar extensors in isolation.

149 The device has high test-retest reliability values of $r=0.81-0.97$ in asymptomatic persons (Graves et al., 150 1990). An intra-class correlation coefficient (ICC) was conducted to assess pre-test MVC test-retest 151 reliability at $72^{\circ}$ between conditions (ICC $=0.931(95 \% \mathrm{Cl}, 0.845-0.972)$ ) supporting the use of $\mathrm{MVC}$ for 152 fatigue response testing in our laboratory.

Fatigue Response Testing

Following the familiarisation session, participants were invited to return to the laboratory on 3 separate occasions (with not less than 72 hours between) where they were assigned each testing condition ( $\mathrm{HL}, \mathrm{LL}$, and $\mathrm{CON}$ ) in a computer generated randomised order. The FRT required participants to complete the previously described testing procedure (pre-MVC) and then complete one of three conditions; a single set of ILEX exercise with a load equating to $80 \%$ of the maximum torque (HL), a single set of ILEX exercise with a load equating to $50 \%$ of the maximum torque (LL) or a control condition where the participants performed no exercise. Exercise was performed at 2 seconds concentric: 4 seconds eccentric (2:4) repetition duration to ensure standardisation with visual time feedback on a display in front of the participant. The ILEX machine also provides an audible sound at the completion of each phase of the repetition (e.g. at full flexion and full extension) to ensure the full range of motion is performed for each repetition. As fatigue incurred, the repetition duration generally increased but participants were encourage never to move faster than the predetermined repetition duration. The exercise was ceased when, despite their maximum effort, participants could not complete the concentric phase of a repetition (e.g. momentary failure; see Steele et al. (2017a) for a detailed description). Immediately ( $<10$ seconds) following cessation of the dynamic exercise the participant 
171 production incurred as a result of the dynamic exercise. FRT testing using the ILEX machine has

172 previously been reported elsewhere (Edinborough et al. 2016).

As a result of pilot testing, a difference was identified for the time-under-load (TUL) between

174 the $\mathrm{HL}$ and LL conditions. As such to ensure parity in time interval ( $\sim 3$ minutes) between the pre- and 175 post-MVC testing following the HL, LL, and CON conditions, the dynamic exercise was delayed by 120

176 and 30 seconds for the $\mathrm{HL}$ and LL conditions, respectively. This rest interval was applied between the

177 pre-MVC and exercise condition so that the post MVC could be performed immediately after the

178 exercise condition. For the CON condition participants remained seated in the ILEX machine for 3

179 minutes before completing the post-MVC.

Immediately following each condition (but before the post-MVC), each participant was asked to report a rating of perceived exertion for effort (RPE-E) and discomfort (RPE-D) using 0-10 scales that permitted appropriate differentiation of the 2 perceptions (Fisher et al., 2017a; 2017b; Steele et al., 183 2017b).

Statistical Analyses considered as the decrease in MVC as a result of the training condition in both absolute (post-MVC Nm pre-MVC Nm) and relative units ([post-MVC Nm / pre-MVC Nm] x 100). The independent variable considered was the exercise condition ( $\mathrm{HL}, \mathrm{LL}$, and CON), as well as sex (male and female), and the dependent variables included pre-MVC (the MVC prior to each condition), absolute and relative fatigue, time under load (TUL), and RPE-D. Analysis was not performed for RPE-E since all participants reported maximal effort (i.e. 10) at the cessation of dynamic exercise at both $\mathrm{HL}$ and $\mathrm{LL}$. distribution and Mauchly's test was used to examine assumptions of sphericity for repeated measures. 
194 For variables collected across all 3 conditions (pre-MVC, absolute, and relative fatigue), data met

195 assumptions of normality and sphericity and thus a $3 \times 2$ repeated measures analysis of variance (ANOVA)

196 was used to compare within participants across the within participant factor 'condition' and between

197 participant factor 'sex'. A $2 \times 2$ repeated measures ANOVA was used in the case of TUL as it was only

198 measured during HL and LL conditions. Post hoc pairwise comparisons with a Bonferroni correction were

199 used for significant effects by 'condition', 'group', or 'condition x group'. RPE-D data did not meet

200 assumptions of normality of distribution and thus a Wilcoxon test was conducted to examine differences

201 between conditions. Mann-Whitney U tests were used to compare dependent variables between sexes.

202 Further, within participant ESs using Cohen's $d$ (Cohen, 1992) were calculated for absolute and

203 relative fatigue for each condition ( $\left.d=\mu_{\text {change }} / \sigma_{\text {change }}\right)$, where an ES of 0.20-0.49 was considered as small,

$2040.50-0.79$ as moderate and $\geq 0.80$ as large. All statistical analyses were performed using IBM SPSS

205 Statistics for Windows (version 23; IBM Corp., Portsmouth, Hampshire, UK) and $p<0.05$ set as the limit

206 for statistical significance.

\section{Results}

Three participants ( 2 female and 1 male) were withdrawn from the study due to non-attendance

of testing sessions leaving 9 males and 8 females for data analysis (participant characteristics are provided in table 1). 1.113, $p=0.342)$, or interaction effects for 'condition $x$ group' $\left(F_{(2,30)}=1.609, p=0.217\right)$, though there was a significant effect by 'group' alone $\left(\mathrm{F}_{(1,15)}=10.663, p=0.005\right.$; estimated marginal means \pm standard error, Males $=373.1 \pm 20.7$ vs Females $=274.3 \pm 22.0$ ). Pre-MVC by condition and sex are shown in table 2. 
0.001), 'group' $\left(F_{(1,15)}=8.104, p=0.012\right)$, and 'condition $x$ group' $\left(F_{(2,30)}=5.248, p=0.011\right)$ for absolute

fatigue. Post hoc pairwise comparisons revealed significant between condition differences between CON and both HL $(p<0.001)$ and LL $(p<0.001)$, and between HL and LL $(p=0.005)$. ESs for absolute fatigue were small or negligible for CON (Males $=0.27$, Females $=-0.09$ ), and large for both HL (Males $=-1.84$, Females $=-0.83$ ), and LL (Males $=-3.11$, Females $=-2.39$ ). Repeated measures ANOVA revealed a

TUL by condition and sex is shown in table 3 . 


\section{Discussion}

The aims of the present study were to compare the fatigue responses of participants performing dynamic ILEX resistance exercise at heavier- and lighter- loads as well as compare these between male and female participants. The findings obtained from this study contribute to the current dearth in the

literature of both sex comparative studies and fatigue following dynamic exercise performed to

245 momentary failure.

Acute Fatigue

While similarly to our previous work (Fisher et al., 2017a) LL conditions resulted in both a higher absolute-, as well as relative- fatigue. Our analyses also revealed that males and females differ in their fatigability in the lumbar extensors. Significantly greater absolute fatigue was incurred by males performing exercise with the same relative load. Although, whilst still greater compared with females, differences in relative fatigue were not significant; possibly as a result of a type II error as previous research has supported sex differences in relative fatigue (Gentil et al., 2017a). In the HL condition males showed a mean decrement in force production of $77.1 \mathrm{Nm}(\sim 21 \%)$ compared to $32.6 \mathrm{Nm}(\sim 11 \%)$ for females, whereas following the LL condition males showed a mean decrease of $128.8 \mathrm{Nm}(\sim 33 \%)$ compared to $70.7 \mathrm{Nm}(\sim 26 \%)$ for females. Correspondingly, endurance capacity appears to differ between males and females, as time-under-load (time to task failure) was shorter for males than

257 females in the LL condition (mean = 133.3 vs. 174.0 seconds, respectively), but not the HL condition 258 (mean $=57.7$ vs. 62.4 seconds, respectively). The present data suggests that, for the lumbar extensors, 259 when exercising to momentary failure using a $\mathrm{HL}(80 \% \mathrm{MVC})$ males and females show similar time to task failure but show a large disparity in their absolute and relative fatigue following exercise. In 
262 exercising for $\sim 41$ seconds ( $24 \%$ ) longer than males, though showed a more similar decrement in force

263 production $($ males $=33 \%$, females $=26 \%$ ).

The present findings agree with the body of literature suggesting women show a smaller decrement in maximal force production compared to males, following fatiguing exercise (Hunter, 2016).

The greater TUL in females compared to males is also supported by studies showing sex-based

discrepancies in endurance capacity during muscular contraction (Maughan et al., 1986; Clark et al., 2003; Gentil et al., 2017a). However, where previous data suggested a similar number of repetitions prior to task failure between males and females for dynamic TEX exercise at 50\%MVC (Clark et al., 2003), our data suggests that where the lumbar extensors are considered in isolation males show a shorter time to failure, and greater degree of fatigue. that males and females show similar time to task failure at high-force or HL exercise (80\% MVC for isometric knee extension; $80 \%$ and $90 \%$ 1-repetition maximum for dynamic elbow flexion). Whilst our data supports this regarding time to task failure (57.7 and 62.4 seconds for males and females, respectively) we have shown that, despite the similar duration of exercise, males incurred a greater absolute and relative degree of fatigue. hypothesised to relate to differences in muscle fibre type (Bajek et al., 2000). Type I muscle fibres have a greater oxidative capacity which makes them markedly more fatigue resistant allowing contractions to be sustained over a longer period but of lower force output than type II muscle fibres. Indeed, our results revealed significant between sex differences in pre-MVC - supportive of greater strength in males compared to females. In addition, because the cross-bridge cycling is faster in type II muscle fibres, 
285

286

287

288

289

290

291

292

293

294

295

296

297

298

299

300

301

302

303

304

305

306

307

308

et al., 2010). Any disparity between HL and LL might be explained by the location of the onset of fatigue in relation to the $\alpha$-motor neuron. Research has suggested that LL activities likely incur elevated central motor output and as such incur higher levels of fatigue and greater reductions in maximal torque. With $\mathrm{HL}$ exercise fatigue appears to develop progressively and exercise cessation occurs as a result of centrally mediated factors (a decrease in the number and discharge of motor units). In contrast, fatigue as a result of $L L$ activities appears to occur peripherally as a result of metabolic changes within the muscle preventing transmission of muscle action potentials (Boyas \& Guével, 2011; Gandevia, 2001).

Research has suggested women have either a greater relative percentage of type I fibres compared to males (Bajek et al., 2000), or have a similar number but show a larger area of type I than type II muscle fibres (70-75\% compared to 54-58\%) when compared to males (Thorstensson \& Carlson, 1987; Mannion et al., 1997). The lumbar extensors are postural muscles and, as such, it would be expected that type I fibres predominate in this group due to a need for sustained or repeated lower force actions. However, this disparity in relative percentage and/or size of type I fibres might explain the greater fatigue resistance in females compared to males observed in the present study, both in strength, the degree of fatigue incurred at both HL and LL, and the time to task failure in LL. However, we should acknowledge that the present study was not intended to assess muscle fibre type disparity or causal factors of fatigue, and as such, we only speculate on potential underpinning mechanisms.

From an applied perspective it is important to recognise the differences in performance and fatigue decrement between males and females. Our data suggests that females do not incur the same degree of fatigue as males following similar exercise protocols, and indeed that females might be able to sustain longer exercise duration at the same relative loads when using low, but not high loads. As such females should manipulate training variables accordingly when training with high loads, performing either greater repetitions at the same relative load, or using heavier relative loads than males. Furthermore, if females do not show the same performance decrement then it might be feasible that 
309 females can train at a higher frequency than males to optimise adaptation. However, it should be noted

310 that in the present paper we have only examined the acute fatigue response. Previous research using a

311 larger training volume (4 sets of 10-repetitions @10-repetition maximum, followed by 4 sets of 10

312 repetitions @ 80\% of 10-repetition maximum) resulted in a similar reduction in peak torque of the

313 elbow flexors between males and females. However, data suggested a longer time to return to baseline

314 torque for females compared to males (Flores et al., 2011). Whilst the relevance to the present study is

315 limited since there is considerable disparity in exercise volume between the studies, future research

316 might consider recovery from fatiguing exercise between males and females using different exercise

317 volumes and across different muscle groups.

\section{Effort and Discomfort}

No analyses were performed for RPE-E since all participants reported maximal values as a result

of training to momentary failure (Steele et al., 2017a). This is supportive of previous research which has

shown that effort and discomfort can be distinguished independently (Fisher et al., 2017b, Steele et al.,

2017b). Performing repetitions to momentary failure in the LL condition induced greater discomfort

where effort and discomfort have been assessed independently (Fisher et al., 2017b). Our analyses also

325 showed no sex-based differences for RPE-D $(p>0.05)$. Previous research has reported varying but

326 submaximal values for effort when assessed alone (Shimano et al., 2006). However, it has previously

327 been discussed that where exercise is continued to the point of momentary failure effort should be

328 maximal irrespective of load, time-under-load, repetitions completed prior to task failure, etc., and that

329 variation in perceptual responses are likely a product of discomfort (Smirnaul, 2012; Steele et al.,

$3302017 \mathrm{~b}$ ). In this sense it is important to be able to anchor effort using the RPE-E and independently assess

331 discomfort (RPE-D), and vice-versa, as we have done so in the present article. 
MacDougall et al., 1999; Takada et al., 2012). Perceptions of discomfort appear to be linked to afferent

feedback and thus a reason why increased metabolic stress may promote greater discomfort during LL motor output (de Morree, Klein \& Marcora, 2012) which may explain the similarly maximal RPE-E that

participants gave for both LL and HL when performed to momentary failure. Indeed, it has been argued being required upon achieving momentary failure. again only speculate upon the potential influences of central or peripheral response. The results from our study may however be useful in a practical sense. Since greater discomfort was incurred in adaptations in muscular strength can occur using both heavier- and lighter- loads of resistance (Assunção et al., 2016; Schoenfeld et al., 2017; Fisher et al., 2017b; 2017c), individuals may be more inclined to exercise using a heavier load since it appears to incur a lower level of discomfort.

\section{Limitations}


355

356

357

sample size for between condition comparisons, only 17 participants completed the study and as such between sex comparisons may have been underpowered (possibly explaining the lack of significant interaction effects for relative fatigue; indeed, post hoc observed $\beta$ for this was 0.318 ). Future work might consider the typical effect size differences between sexes in determining sample estimates, and also might consider what constitutes a clinically meaningful difference. Further, our results may be limited in application to persons with the characteristics that conformed to our inclusion criteria. Whilst we have discussed the efficacy of strengthening the lumbar extensors to reduce CLBP there were no participants involved in the study with the condition. As such it would be unreasonable to assume these findings would be equated with this particular population, therefore it may be beneficial to replicate this study using both CLBP symptomatic and asymptomatic individuals.

\section{Conclusion}

The present study advances the limited research in acute responses of fatiguing exercise to differing loading strategies, notably comparing males and females, and assessing the lumbar extensors in isolation. The findings revealed load dependent disparities, in addition to sex based differences, in fatigue response as a result of ILEX exercise performed to momentary failure. Since females do not incur the same degree of fatigue as males following similar exercise protocols, females might manipulate training variables accordingly. Albeit speculative, it might be more efficacious for females to perform a larger number of repetitions for the same relative load, or use a greater relative training load compared to males to increase volume-load or maximise strength-specific adaptations. Furthermore, if a lesser decrement in performance is incurred from a similar training volume then females might consider either a). Increased training volume, or b). Greater training frequency, both of which might result in greater calorific expenditure which might favourably optimise body composition adaptations. However, the latter is recommended tentatively as the differences between sexes in rate of recovery to baseline after $\mathrm{RT}$ is not clear. 

resistance exercise (Assunção et al., 2016; Schoenfeld et al., 2017; Fisher et al., 2017b; 2017c) and as such our consideration of discomfort relating to load is important. Indeed, previous research has shown that low-volume (1 day / week) ILEX resistance exercise can strengthen the lumbar extensors (Fisher et al., 2013) and as a result, reduce CLBP (Bruce-Low et al., 2012; Steele et al., 2015a). Lighter load exercise is often prescribed in rehabilitation settings perhaps owing to clinician's perceptions that it may be less likely to result in pain. Indeed, clinicians in general seem reluctant to recommend high intensity of effort exercise either (Munneke et al., 2004). However, in addition to exercise related discomfort, group III/IV muscle afferents are involved in nociception and thus may be involved in perception of pain (McCord \& Kaufman, 2010). It seems prudent for clinicians to know firstly that, effort and load are not synonymous and, given correct instruction, people can differentiate effort and discomfort; and secondly that lighter load exercise might be contraindicated due to the increased fatigue and discomfort associated with it.

Discomfort associated with LL exercise might be misinterpreted by a patient as pain relating to their injury. However, tools exist allowing patients to differentiate the qualities of different pain experiences (Melzack and Katz, 2001) and so it may be the case that with correct instruction patients can differentiate exercise related perceptions of discomfort from perceptions of pain associated with their injury. Future research might consider the use of $\mathrm{HL}$ and $\mathrm{LL}$ resistance exercise in the lumbar extensors in rehabilitation environments to assess the efficacy of the load-based training prescription with careful consideration to the perceptual elements of effort, exercise related discomfort, and injury related pain. 
398

399

400

401

402

403

404

405

406

407

408

409

410

411

412

413

414

415

416

417

418

419

420

421

422

423

424

425

\section{References}

Assunção, A. R., Bottaro, M., Ferreira-Junior, J. B., Izquierdo, M., Cadore, E. L., and Gentil, P. 2016. The Chronic Effects of Low- and High-Intensity Resistance Training on Muscular Fitness in Adolescents. PLOS ONE 11(8): e0160650.

Bajek, S., Bobinac, D., Bajek, G., Vranić, T. S., Lah, B., Dragojević, D. M. 2000. Muscle fiber type distribution in multifidus muscle in cases of lumbar disc herniation. Acta Medica Okayama 54(6):235-241.

Boyas, S., Guével, A. 2011. Neuromuscular fatigue in healthy muscle: underlying factors and adaptation mechanisms. Annals of Physical and Rehabilitation Medicine 54:88-108.

Bruce-Low, S., Smith, D., Bissell, G., Burnet, S., Fisher, J., and Webster, L. 2012. One lumbar extension training session per week is sufficient for strength gains and reductions in pain in patients with chronic low back pain ergonomics. Ergonomics 55(4);500-507.

Clark, B. C., Manini, T. M., Thé, D. J., Doldo, N. A., Ploutz-Snyder, L. L. 2003. Gender differences in skeletal muscle fatigability are related to contraction type and EMG spectral compression. Journal of Applied Physiology 94: 2263-2272.

Cohen, J. 1992. A power primer. Psychological bulletin 112(1):155-159.

Conway, R., Behennah, J., Fisher, J., Osborne, N. and Steele, J. 2016. Associations between Trunk Extension Endurance and Isolated Lumbar Extension Strength in Both Asymptomatic Participants and Those with Chronic Low Back Pain. Healthcare 4(3):70.

de Morree, H.M., Klein, C., Marcora, S. M. 2012. Perception of effort reflects central motor command during movement execution. Psychophysiology 49(9):1242-1253.

Edinborough, L., Fisher, J. P., Steele, J. 2016. A comparison of the effect of kettlebell swings and isolated lumbar extension training on acute torque production of the lumbar extensors. Journal of Strength and Conditioning Research 30(5):1189-1195.

Enoka, R. M., Stuart, D. G. 1992. Neurobiology of muscle fatigue. Journal of Applied Physiology 72: 163148.

Faul, F., Erdfelder, E., Buchner, A., Lang, A. G. 2009. Statistical power analysis using G*Power 3.1: tests for correlation and regression analyses. Behavior Research Methods 41:1149-1160. 
426 Faul, F., Erdfelder, E., Lang, A. G., Buchner, A. 2007. G*Power: a flexible statistical power analysis

427

428

429

430

431

432

433

434

435

436

437

438

439

440

441

442

443

444

445

446

447

448

449

450

451

452

453 program for the social, behavioural and biomedical sciences. Behavior Research Methods 39:175191.

Fisher, J. P., Farrow, J., Steele, J. 2017a. Acute fatigue, and perceptual responses to resistance exercise. Muscle and Nerve 56:E141-E146

Fisher, J. P., Ironside, M., Steele, J. 2017b. Heavier- and lighter-load resistance training to momentary failure produce similar increases in strength with differing degrees of discomfort. Muscle and Nerve 56(4):797-803.

Fisher, J., Bruce-Low, S. and Smith, D. 2013. A randomized trial to consider the effect of Romanian deadlift exercise on the development of lumbar extension strength. Physical Therapy in Sport 14(3):139-145.

Fisher, J., Steele, J., Smith, D. 2017c. High- and Low-Load Resistance Training: Interpretation and Practical Application of Current Research Findings. Sports Medicine 47(3):393-400.

Flores, D. F., Gentil, P., Brown, L. E., Pinto, R. S., Carregaro, R. L., Bottaro, M. 2011. Dissociated time course of recovery between genders after resistance exercise. Journal of Strength and Conditioning Research 25(11):3039-3044.

Gandevia, S. C. 2001. Spinal and supraspinal factors in human muscle fatigue. Physiological Reviews 81:1725-1789.

Gentil, P., Campos, M.H., Soares, S., De Conti Teixeira Costa, G., Paoli, A., Bianco, A., Bottaro, M. $2017 a$. Comparison of elbow flexor isokinetic peak torque and fatigue index between men and women of different training level. European Journal of Translational Myology 27(4):246-250.

Gentil, P., Fisher, J., and Steele, J. 2017b. A Review of the Acute Effects and Long-Term Adaptations of Single- and Multi-Joint Exercises during Resistance Training. Sports Medicine 47(5): 843-855.

Graves, J. E., Pollock, M. L., Carpenter, D. M., Leggett, S. H., Jones, A., MacMillan, M., Fulton, M. 1990. Quantitative assessment of full range of motion isometric lumbar extension strength. Spine 15(4):289-294.

Hunter, S. K. 2016. The relevance of sex differences in performance fatigability. Medicine and Science in Sports and Exercise 48:2247-2256. 
454

455

456

457

458

459

460

461

462

463

464

465

466

467

468

469

470

471

472

473

474

475

476

477

478

479

480

Hunter, S. K., Critchlow, A., Shin, I., Enoka, R. M. 2004. Fatigability of the elbow flexor muscles for a sustained submaximal contraction is similar in men and women matched for strength. Journal of Applied Physiology 96:195-202.

MacDougall, J. D., Ray, S., Sale, D.G., McCartney, N., Lee, P., Garner, S. 1999. Muscle substrate utilization and lactate production during weight lifting. Canadian Journal of Applied Physiology 24:209-215.

Mannion, A. F., Dumas, G. A., Cooper, R. G., Espinosa, F. J., Faris, M. W., Stevenson, J. M. 1997. Muscle fibre size and type distribution in thoracic and lumbar regions of erector spinae in healthy subjects without low back pain: normal values and sex differences. Journal of Anatomy 190(Pt4):505-513.

Marcora, S. 2009. Perception of effort during exercise is independent of afferent feedback from skeletal muscles, heart, and lungs. Journal of Applied Physiology 106:2060-2062.

Maughan, R. J., Harmon, M., Leiper, J. B., Sale, D., Delman, A. 1986. Endurance capacity of untrained males and females in isometric and dynamic muscular contractions. European Journal of applied physiology and occupational physiology 55(4):395-400

McCord, J. L., Kaufman, M. P. 2010. Chapter 12. Reflex autonomic responses evoked by group III and IV muscle afferents. In Kruger, L., Light, A. R. eds. Translational Pain Research: From Mouse to Man. Boca Raton, FL: Taylor and Francis

Melzack, R., Katz, J. 2001. The McGill Pain Questionnaire: Appraisal and current status. In Turk, D. C., Melzack, R. eds. Handbook of pain assessment. New York, NY, US: Guilford Press:

Morton, R. W., McGlory, C., Phillips, S. M. 2015. Nutritional interventions to augment resistance training-induced skeletal muscle hypertrophy. Frontiers in Physiology 6:1-9.

Munneke, M., de Jong, Z., Zwinderman, A. H., Ronday, H. K., van den Ende, C. H. M., Vliet Vlieland T. P. M., Hazes, J. M. W. 2004. High intensity exercise or conventional exercise for patients with rheumatoid arthritis? Outcome expectations of patients, rheumatologists, and physiotherapists. Annals of the Rheumatic Diseases 63:804-808

Schoenfeld, B. J., Grgic, J., Ogborn, D., Krieger, J. W. 2017. Strength and hypertrophy adaptations between low-versus high-load resistance training: A Systematic review and meta-analysis. Journal of Strength and Conditioning Research 31(12):3508-3523. 
481 482 483 484 485 486

Schott, J., McCully, K., Rutherford, O.M. 1995. The role of metabolites in strength training II. Short versus long isometric contractions. European Journal of Applied Physiology 71:337-341.

Shimano T, Kraemer WJ, Spiering BA, Volek JS, Hatfield DL, Silvestre R, Vingren JL, Fragala MS, Maresh CM, Fleck SJ, Newton RU, Spreuwenberg LP, Häkkinen K. 2006. Relationship between the number of repetitions and selected percentages of one repetition maximum in free weight exercises in trained and untrained men. Journal of Strength and Conditioning Research 20:819-823.

Smirnaul, B. D. P. C. 2012. Sense of effort and other unpleasant sensations during exercise: clarifying concepts and mechanisms. British Journal of Sports Medicine 46:308-311.

Steele, J., Bruce-Low, S. and Smith, D. 2013. A review of the specificity of exercises designed for conditioning the lumbar extensors. British Journal of Sports Medicine 49(5):291-297.

Steele, J., Bruce-Low, S. Smith, D. 2014. A reappraisal of the deconditioning hypothesis in low back pain: review of evidence from a triumvirate of research methods on specific lumbar extensor deconditioning. Current Medical Research and Opinion 30(5):865-911.

Steele, J., Bruce-Low, S., Smith, D. 2015a. A review of the clinical value of isolated lumbar extension resistance training for chronic low back pain. Physical Medicine and Rehabilitation 7(2):169-187.

Steele, J., Fisher, J., Giessing, J., Gentil, P. 2017a. Clarity in Reporting Terminology and Definitions of Set End Points in Resistance Training. Muscle and Nerve 56(3):368-374.

Steele, J., Fisher, J., McKinnon, S., McKinnon, P. 2017b. Differentiation between perceived effort and discomfort during resistance training: reliability of trainee ratings of effort and discomfort, and reliability and validity of trainer ratings of trainee effort. Journal of Trainology 5:1-8.

Steele, J., Fitzpatrick, A., Bruce-Low, S., Fisher, J. 2015b. The effects of set volume during isolated lumbar extension resistance training in recreationally trained males. PeerJ 3:e878.

Takada, S., Okita, K., Suga, T., Omokawa, M., Kadoguchi, T., Sato, T., et al. 2012. Low-intensity exercise can increase muscle mass and strength proportionally to enhance metabolic stress under ischemic conditions. Journal of Applied Physiology 113:199-205.

Thorstensson, A., Carlson, H. 1987. Fibre types in human lumbar back muscle. Acta Physiologcial Scandinavica 131(2):195-202. 
508 Westerblad, H., Bruton, J. D., Katz, A. 2010. Skeletal muscle: Energy metabolism, fiber types, fatigue, and 509 adaptability. Experimental Cell Research 316:3093-3099. 
Figure 1

Restraint system for the MedX Isolated lumbar extension machine

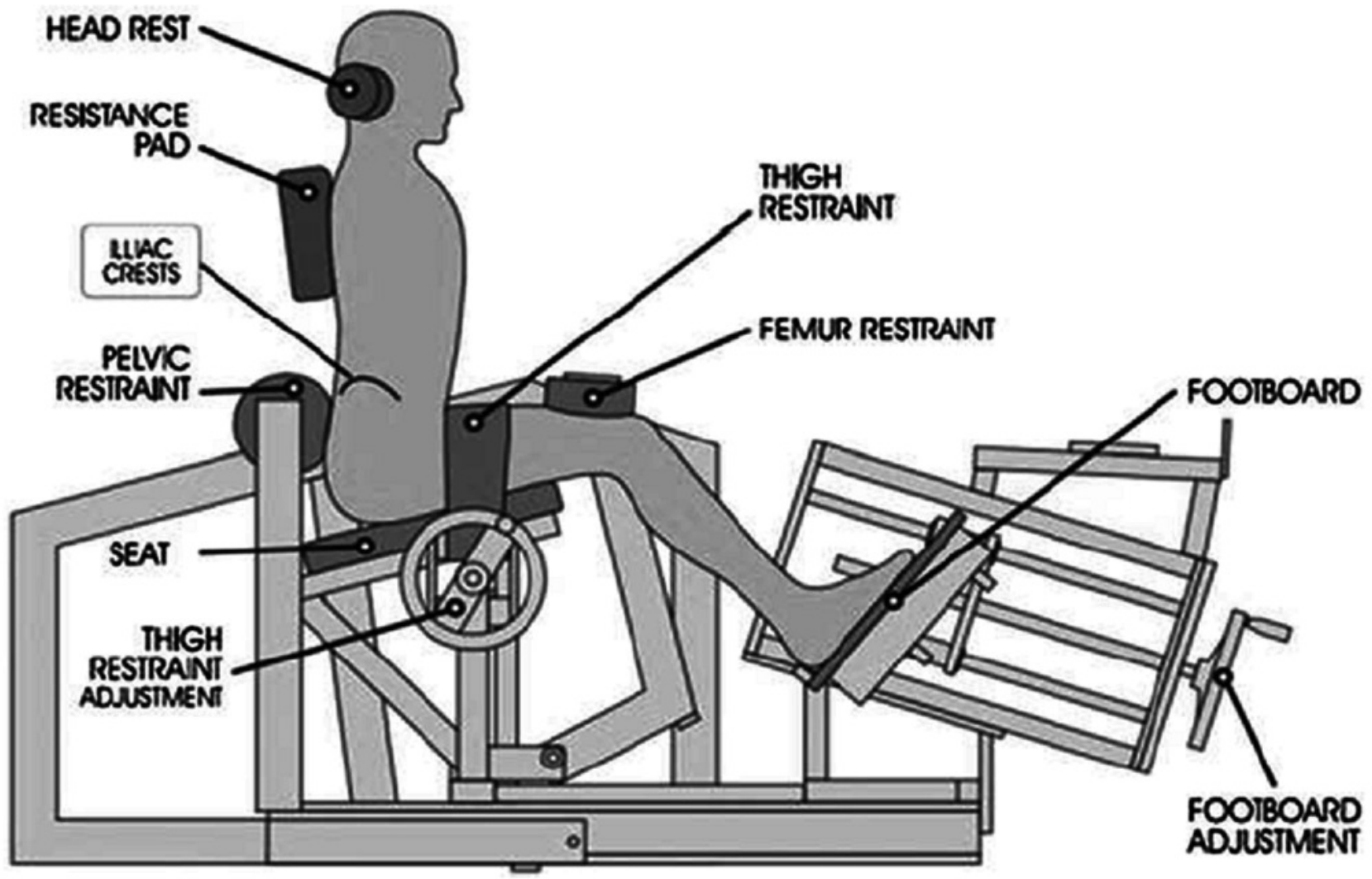


Figure 2

Mean $\pm 95 \% \mathrm{Cls}(\mathrm{A})$ and individual responses (B) for absolute fatigue, and mean $\pm 95 \% \mathrm{Cls}$ (C) and individual responses (D) for relative fatigue, between conditions and sexes

A)

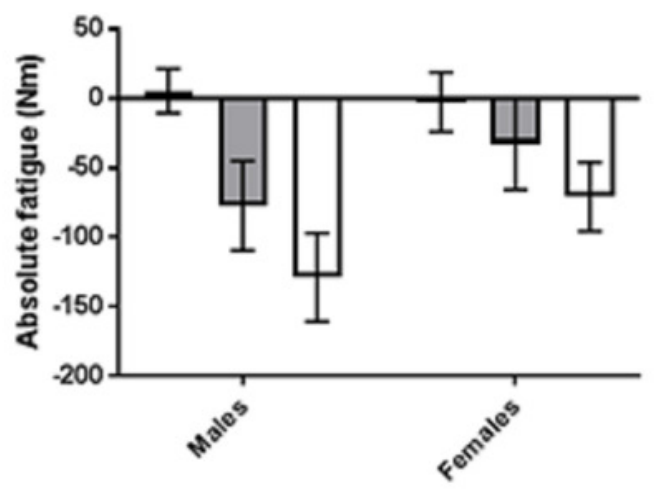

C)

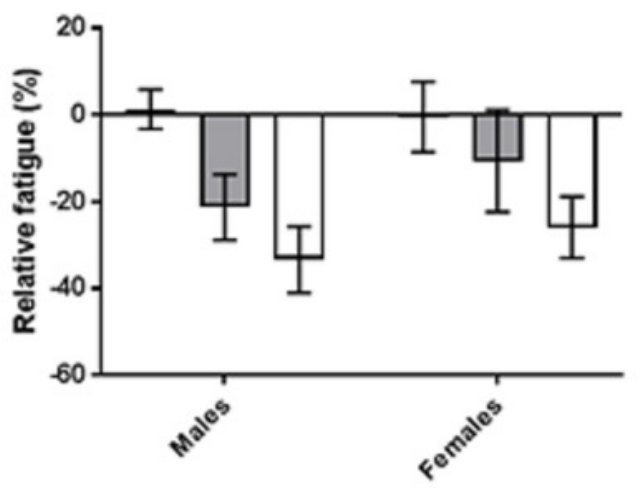

B)
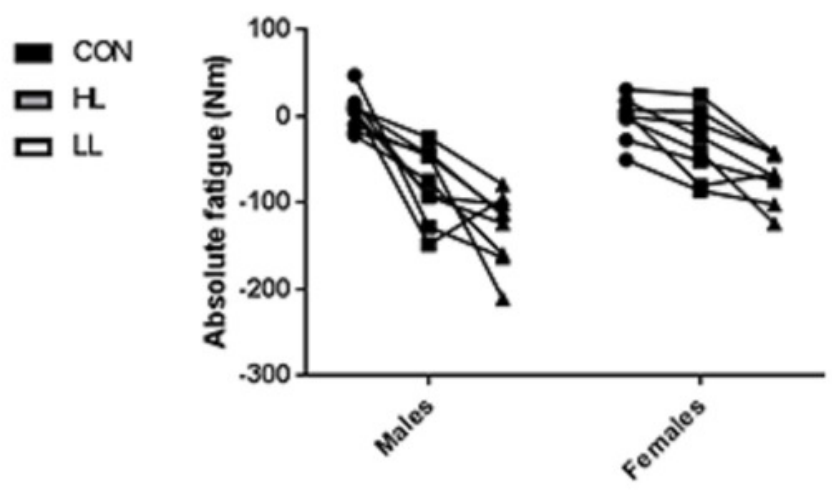

D)

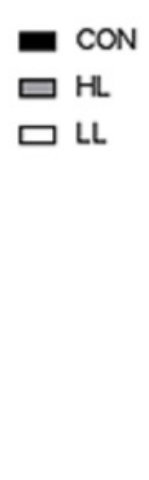

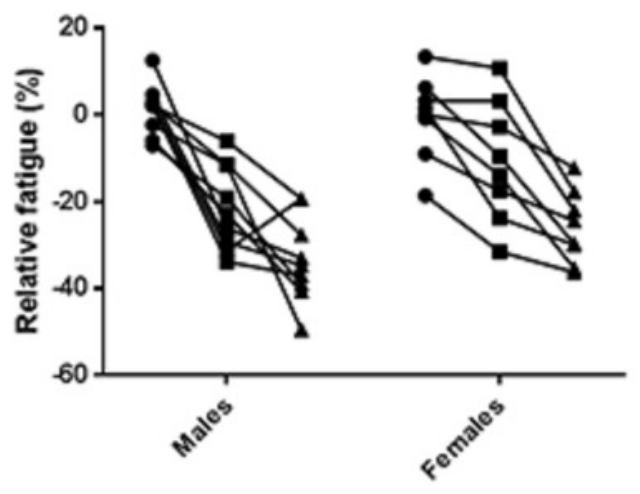


Table $\mathbf{1}$ (on next page)

Participant Characteristics (mean \pm SD) 
1

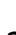

2

3 Males $(n=9)$

Females $(n=8)$

4

\begin{tabular}{cccc} 
Group & Age (years) & Height $(\mathrm{cm})$ & Weight $(\mathrm{kg})$ \\
\hline Males $(n=9)$ & $23.8 \pm 6.4$ & $176.7 \pm 6.2$ & $73.9 \pm 9.3$ \\
Females $(n=8)$ & $21.3 \pm 0.9$ & $170.5 \pm 6.1$ & $65.5 \pm 10.8$
\end{tabular}

5 Characteristics (mean \pm SD)

6

7

8

9

10

7

8
Table

1.

Particip

ant 


\section{Table 2 (on next page)}

Mean $( \pm \mathrm{SD}$ ) values for time-under-load between sex and condition 


\begin{tabular}{|c|c|c|c|c|}
\hline Condition & Sex & $\begin{array}{c}\text { Pre-MVC } \\
(\mathrm{Nm} ; \text { mean } \pm \mathrm{SD})\end{array}$ & $\begin{array}{l}\text { Absolute change in } \\
\text { MVC } \\
(\mathrm{Nm} \text {; mean } \pm \mathrm{SD})\end{array}$ & $\begin{array}{l}\text { Relative change in } \\
\text { MVC } \\
\text { (\%; mean } \pm \text { SD) }\end{array}$ \\
\hline \multirow[t]{2}{*}{ CON } & Males & $355.2 \pm 56.4$ & $5.59 \pm 20.7$ & $1.3 \pm 5.9$ \\
\hline & Females & $277.4 \pm 45.4$ & $-2.55 \pm 25.5$ & $-0.5 \pm 9.7$ \\
\hline \multirow[t]{2}{*}{$\mathrm{HL}$} & Males & $369.6 \pm 97.2$ & $-77.1 \pm 41.9$ & $-21.3 \pm 9.8$ \\
\hline & Females & $272.3 \pm 58.5$ & $-32.6 \pm 39.4$ & $-10.6 \pm 14.0$ \\
\hline \multirow[t]{2}{*}{ LL } & Males & $394.4 \pm 78.7$ & $-128.8 \pm 41.4$ & $-33.3 \pm 9.9$ \\
\hline & Females & $273.2 \pm 57.0$ & $-70.7 \pm 29.6$ & $-25.9 \pm 8.4$ \\
\hline
\end{tabular}

1 Table 2. Mean pre-MVC, absolute fatigue, and relative fatigue within groups and load conditions.

$2 \mathrm{MVC}=$ maximal voluntary contraction; $\mathrm{CON}=$ control condition; $\mathrm{HL}=$ heavier-load condition; $\mathrm{LL}=$ lighter3 load condition; $\mathrm{Nm}=$ newton metres 


\section{Table 3(on next page)}

Mean pre-MVC, absolute fatigue, and relative fatigue within groups and load conditions $M V C=$ maximal voluntary contraction; $\mathrm{CON}=$ control condition; $\mathrm{HL}=$ heavier-load condition; $\mathrm{LL}=$ lighter-load condition; $\mathrm{Nm}=$ newton metres 
1 Table 3. Mean ( \pm SD) values for time-under-load between sex and condition

\begin{tabular}{lcc}
\hline & Heavier-load (seconds) & Lighter-load (seconds) \\
\hline Males & $57.7 \pm 14.2$ & $133.3 \pm 29.7$ \\
Females & $62.4 \pm 17.6$ & $174.0 \pm 54.5$ \\
\hline
\end{tabular}

2

3 


\section{Table 4(on next page)}

Mean $( \pm$ SD) values for discomfort (RPE-D) between sex and condition 
1 Table 4. Mean ( $\pm S D$ ) values for discomfort (RPE-D) between sex and condition

\begin{tabular}{lcc}
\hline & Heavier-load & Lighter-load \\
\hline Males & $6.33 \pm 0.71$ & $8.00 \pm 0.71$ \\
Females & $6.25 \pm 0.71$ & $8.25 \pm 0.71$ \\
\hline
\end{tabular}

2

3 